\title{
Dehydration of D-mannitol: building block for C-nucleoside synthesis
}

\section{Edmilson Clarindo de Siqueira, ${ }^{a}$ Alexander Y.Nazarenko ${ }^{b}$ and Bogdan Doboszewski ${ }^{*}$}

Departamento de Química, Universidade Federal Rural de Pernambuco,52171-900 Recife, PE, Brasil, ${ }^{a}$ Chemistry Department, State University of New York, College at Buffalo, 1300 Elmwood Ave; Buffalo, NY 14222-1095, USA

*bdoboszewski@hotmail.com

Keywords: D-mannitol, cyclization, $x$-ray

\section{INTRODUCTION}

D-Mannitol $\mathbf{1}$ is a convenient starting material for organic synthesis because of the homotopic relation between the upper and the lower part of the molecule. For this reason dehydration of 1 via 2-5 hydroxyl groups yields one product only, i.e. 2,5,anhydro-D-glucitol 2 . Our interest in 2 and its derivative $\mathbf{5}$ stems from a possibility of application of the latter for C-nucleoside synthesis. Acid catalyzed dehydration of $\mathbf{1}$ was performed followed by some manipulations, and permitted isolation of the 2,5anhydro-4,6-di-O-benzyl-D-glucitol 5, together with 2,6-anhydro-1,3-di-O-benzyl-D-mannitol 7 (or 1,5anhydro-4,6-di-O-benzyl-D-mannitol due to a symmetry of $\mathbf{1}$ ). A structure of $\mathbf{7}$ was confirmed by $x$ ray analysis.

\section{RESULTS AND DISCUSSION}

Acid-catalyzed dehydration of 1 was realized following patented procedure ${ }^{1}$ which we modified to facilitate isolation of the products on a smaller scale. $^{2}$ Modification is to subject a crude dehydration mixture to isopropylidenation, and to isolate the main product, 1,3-isopropylidene-D-glucitol, via vacuum dry chromatography, instead of high vacuum distillation. ${ }^{1}$ Further operations shown in the Scheme 2 permitted isolation of the necessary derivative 5 . In some preparations we obtained more polar and highly crystalline compound 7 , whose $x$ ray structure is shown in Picture 1.

Application of $\mathbf{5}$ to get C-nucleoside analogs will be published in due course.

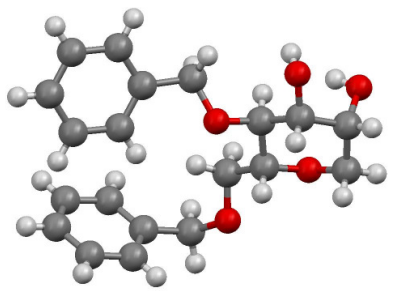

Picture 1. X-Ray structure of 7

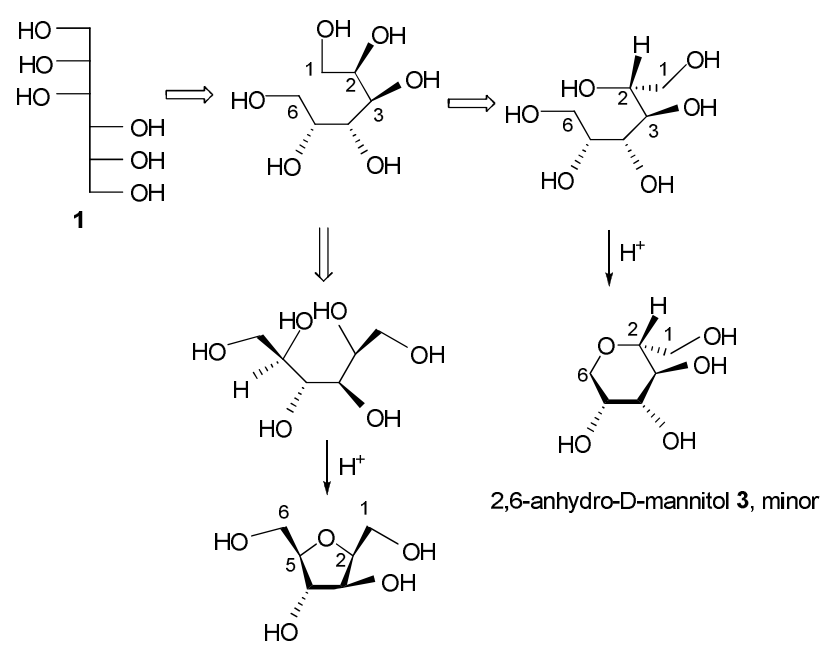

2,5-anhydro-D-glucitol 2, major

Scheme 1. Acid-catalysed dehydration of D-mannitol

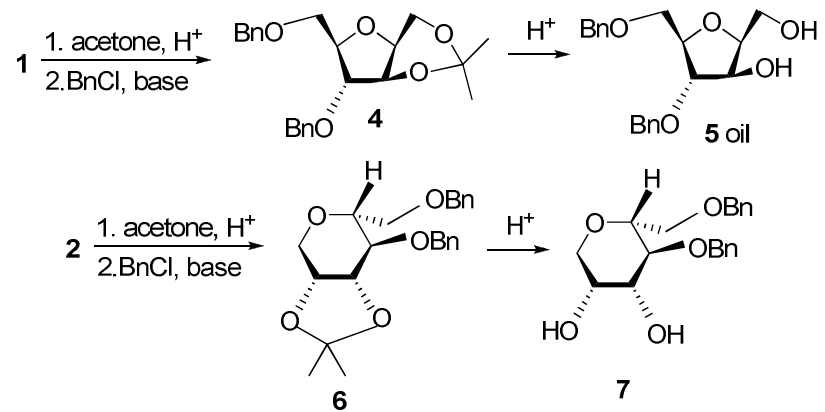

Scheme 2..Synthesis of di-O-benzylated derivative $\mathbf{5}$ and 7.

\section{CONCLUSION}

Synthesis of dibenzylated derivative of D-glucitol $\mathbf{5}$ and of D-mannitol 7 was achieved.

\section{REFERENCES}

${ }^{1}$ Hartman, L. Separation of hexitans (anhydrohexitols). 1970, US Patent $3,484,459$.

${ }^{2}$ Doboszewski, B.; Siqueira, E.C. de. Synth.Commun. 2010, 40, 744

${ }^{3}$ Harwood, L.M. Aldrichim. Acta, 1985, 18, 25. 